\title{
Ostwald Ripening in Rarefied Systems
}

\author{
Victor M. Burlakov \\ Department of Materials, University of Oxford, Parks Road, Oxford OX1 3PH, United Kingdom, \\ and Institute for Spectroscopy Russian Academy of Sciences, Troitsk, Moscow region, 142090, Russia
}

(Received 8 May 2006; published 10 October 2006)

\begin{abstract}
Mass exchange between spherical molecular clusters is analyzed in the case when the mean free path of molecules in the intercluster space is much larger than the cluster sizes but much smaller than average intercluster separation. It is shown that there is a steady-state regime when big clusters grow at the expense of the smaller ones (Ostwald ripening), which is characterized by a one-parametric family of self-similar cluster size distribution functions and by an exponentially growing average cluster size. The obtained selfsimilar cluster size distributions are entirely different from those given by the classical theory of Ostwald ripening. Implications of the obtained results are discussed.
\end{abstract}

DOI: 10.1103/PhysRevLett.97.155703

Microstructure coarsening, or Ostwald ripening (OR) [1], is a phenomenon often observed at the late stages of many first order phase transformations in two-phase mixtures [2], binary alloys [3], clusters on surfaces [4], oilwater emulsions [5], and during epitaxial growth [6,7] and synthesis of nanoparticles [8]. During OR, small clusters of atoms/molecules dissolve, transferring their mass to bigger clusters. Classical mean-field treatment of OR [9-14] predicts a steady-state growth regime with the average cluster size $\langle R\rangle$ increasing in time as $\langle R\rangle \sim t^{\alpha}(1>\alpha>0)$, which is confirmed in numerical $[8,15-19]$ and experimental [47,20-24] investigations of the systems satisfying the theoretical assumptions. All theoretical studies of OR performed so far are confined to the case when the mean free path of molecules in a vapor/solution is small compared to the minimum cluster size. Here we show that OR in a rarefied system, which has a mean free path much larger than the maximum cluster size, deviates significantly from the classical picture: The average cluster size grows exponentially, and clusters tend to have a boxcar size distribution rather than the one described by a LifshitzSlezov [10] or Wagner [11] function.

Classical Lifshitz-Slezov-Wagner (LSW) theory [9-11], as well as all other theories of OR [12-19], is based on an assumption about a local equilibrium between molecular clusters and the surrounding solution, which allows the concentration of molecules at the cluster-solution interface to be obtained using the Gibbs-Thomson relation [25]. In reality, each molecule emitted by a cluster travels a certain distance away from it before reaching equilibrium with the surrounding solution. Local equilibrium is achieved within the Knudsen layer (or rarefied space), which typically has a thickness of approximately one mean free path [26]. In all previous theories of OR, therefore, it is implicit that the molecular mean free path $\lambda$ is negligible compared to the minimum cluster radius $R_{\min }$, which is often not the case for many liquid (solid)-vapor systems. Such systems are realized during gas-phase production of nanoparticulate powders [27], where the OR phenomenon is believed to be involved (see discussion in Ref. [28]). Understanding
PACS numbers: 64.60.My, 05.70.-a

the role of the molecular mean free path in OR is therefore of both theoretical and practical interest.

To illustrate the role of a finite $\lambda$ in OR, we apply the methodology of kinetic gas theory to a conservative system of spherical molecular clusters in the vapor using approximations of classical LSW theory: The total cluster volume is (a) conserved, suggesting that the vapor density is either constant or negligibly low, and (b) negligible compared to the total volume of the system. We consider interfacelimited OR [11] assuming that cluster size evolution is controlled by molecular emission from the cluster surfaces. A cluster of radius $R$ emits a molecular flux $I(R)$ and absorbs a flux $4 \pi R^{2} J_{\rho}$ that is proportional to the average flux density $J_{\rho}$ in the intercluster space. For simplicity, we assume that the clusters absorb every molecule touching their surfaces, although the approach could be formulated in more general terms. Because of conservation of the total cluster volume, the total emitted flux at any time $t$ is equal to the total absorbed flux, namely,

$$
\begin{aligned}
\int_{R_{\min }}^{R_{\max }} I(R) f(R, t) d R & =J_{\rho} \int_{R_{\min }}^{R_{\max }} 4 \pi R^{2} f(R, t) d R, \\
\int_{R_{\min }}^{R_{\max }} f(R, t) d R & =n(t),
\end{aligned}
$$

where the function $f(R, t)$ describes the instantaneous cluster radius distribution (CRD), $n(t)$ is the total number of clusters, and $R_{\min } \approx 0$ and $R_{\max }$ are the minimum and the maximum cluster radii, respectively. Volume balance for a single cluster of radius $R$ implies that

$$
\frac{d R}{d t}=\frac{v}{4}\left(\frac{\int_{0}^{R_{\max }} I(x) f(x, t) d x}{\int_{0}^{R_{\max }} \pi x^{2} f(x, t) d x}-\frac{I(R)}{\pi R^{2}}\right),
$$

where $\nu$ is the volume associated with one molecule.

Molecules emitted from the clusters have a certain probability $P(R, \lambda)$ to return to the cluster of origin due to collisions with other molecules. Taking into account such a probability, the net flux $I(R)$ emitted by the cluster may be written as 


$$
I(R)=K 4 \pi R^{2}[1-P(R, \lambda)],
$$

where $K$ is the molecular emission rate per unit surface area. If the dynamics and binding energy of molecules on the cluster surfaces are not significantly influenced by the surface curvature, $K$ can be considered as independent of $R$, the assumption similar to the $R$-independent interface transfer rate in Wagner theory [11]. This assumption is plausible for large enough clusters (see also the analysis in Ref. [29]), in which the fraction of molecules on the surface is small compared to that in the bulk. The mass fraction of smaller clusters, for which $K$ could be dependent on $R$, is vanishing during ripening and can be neglected. Equations (2) and (3) are the governing equations describing the cluster system evolution for any values of $R$ and $\lambda$. According to these equations, no ripening occurs for $P(R, \lambda)=0$. The value of $P(R, \lambda)$ depends on the parameters determining the regime of mass transport, such as the molecular mean free path $\lambda$, cluster radius $R$, and average intercluster distance $\Lambda$. Assuming $\lambda$ to be constant, e.g., set by a buffer gas density, we analyze OR in the rarefied gas regime of mass transport when $\Lambda \gg \lambda \gg R$.

Any emitted molecule can return to the cluster of origin after $1,2, \ldots k$ collisions with other molecules, suggesting that $P(R, \lambda)=\sum_{k=1}^{\infty} P_{k}(R, \lambda)$. In a simplified picture, the emitted molecules travel away from the cluster along the radius, and the single-collision return probability $P_{1}(R, \lambda)$ can be represented as

$$
P_{1}(R, \lambda)=\int_{0}^{\infty} T(x, \lambda)\left(\int_{x}^{\infty} T\left(x_{1}, \lambda\right) d x_{1}\right) G(x) d x,
$$

where $T(x, \lambda)=\lambda^{-1} \exp (-x / \lambda)$ is the probability density, the integral of which from " 0 " to " $x$ " defines the probability for a molecule to have a collision after traveling the distance $x ; G(x) \simeq 0.5[R /(R+x)]^{2}$ is a geometrical factor defining the probability for a molecule colliding at a distance $x$ from the cluster to scatter along the directions crossing the cluster cross section. A direct calculation using Eq. (4) gives $P_{1}(R, \lambda) \simeq 0.5 R / \lambda$, which is slightly higher than the value obtained taking into account isotropic emission of molecules and the cluster sphericity

$$
P_{1}(R, \lambda) \simeq 0.35 R / \lambda .
$$

We show that for low enough $R / \lambda$ values the total return probability $P(R, \lambda)$ can be approximated by the singlecollision return probability given by Eq. (5). The twocollision return probability is estimated by direct calculation within the same approximations as those used in Eq. (4), giving $P_{2}(R, \lambda) \simeq 5(R / \lambda)^{2}$. Similarly, one can estimate the three-collision return probability $P_{3}(R, \lambda) \simeq$ $0.5(R / \lambda)^{2}$. To estimate higher order multicollision return probabilities, we recall that the mean square displacement of the emitted molecule after $k$ collisions is $\left\langle x^{2}\right\rangle=\lambda^{2} k$. Using this, $P_{k}(R, \lambda)$ can be estimated as $P_{k}(R, \lambda) \approx$ $\left(R^{2} / \lambda^{2}\right)[\exp (-\sqrt{k}) / k]$. Summing up all multicollision return probabilities, one finds that the result does not exceed $6(R / \lambda)^{2}$, i.e., at low enough $R / l$ values, all multicollision contributions to the total return probability can be neglected.

The equation for the time evolution of cluster radii can then be derived by substituting Eq. (3) into Eq. (2) and replacing the return probability in Eq. (3) with the singlecollision return probability given by Eq. (5). It takes the form

$$
\frac{d R}{d t}=\frac{0.35 \nu K}{\lambda}\left[R-R_{c}(t)\right],
$$

where $\quad R_{c}(t)=\left\langle R^{3}\right\rangle /\left\langle R^{2}\right\rangle \quad$ and $\quad\left\langle R^{m}\right\rangle=1 / n(t) \times$ $\int_{0}^{R_{\max }} R^{m} f(R, t) d R \quad(m=2,3)$. According to Eq. (6), all clusters with a radius smaller than $R_{c}(t)$ decrease with time, while those with a radius bigger than $R_{c}(t)$ grow.

As in classical theory of OR, the cluster evolution governed by Eq. (6) possesses a self-similar regime [30] characterized by a scaling invariant CRD function $f(R, t)$

$$
f(R, t)=n_{0} \varphi^{-4}(t) g\left(\frac{R}{\varphi(t)}\right), \quad \int_{0}^{x_{\max }} g(x) d x=1,
$$

where $n_{0}$ is the number of clusters at the onset of the selfsimilar regime. The function $\varphi(t)$ is a time-dependent scaling factor for the range of variation of $R$, and it determines the time dependence of the average cluster radius $\langle R\rangle$. This scaling factor can be obtained from Eq. (6) by substituting $R=R_{\max }^{0} \varphi(t)$ and $R_{c}(t)=R_{c}^{0} \phi(t)$. We find that the exponential function $\varphi(t)=\exp (\beta t)$, with $\beta=$ $0.35 \nu K\left(R_{\max }^{0}-R_{c}^{0}\right) /\left(\lambda R_{\max }^{0}\right)$, is a solution.

The function $f(R, t)$ can now be determined from the continuity equation in the space of cluster sizes [9-11]

$$
\frac{\partial f(R, t)}{\partial t}+\frac{\partial}{\partial t}\left(f(R, t) \frac{d R}{d t}\right)=0 .
$$

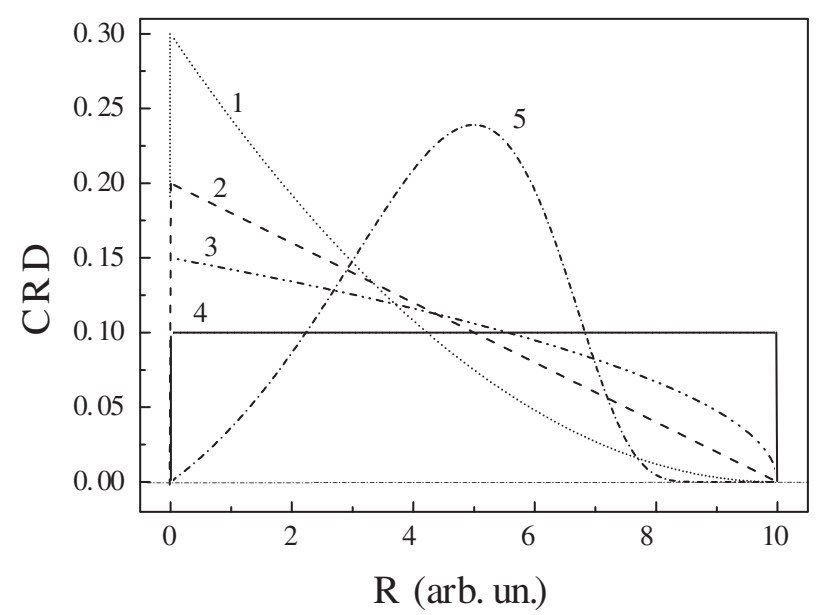

FIG. 1. CRDs in a rarefied system defined in the interval $10 \geq$ $R \geq 0$ and described by Eq. (9) with $d=2.0$ (1), 1.0 (2), 0.5 (3), and 0.0 (4). The latter is the so-called boxcar distribution. Curve 5 shows one of the self-similar distributions obtained in the classical theory of interface-controlled OR $g(x)=$ const. $x x_{\max }^{5}\left(x_{\max }-x\right)^{-5} \exp \left[-3 x /\left(x_{\max }-x\right)\right]$ (Wagner distribution [11]). All CRDs are normalized on " 1 ." 
We see that this has a self-similar solution of the form

$$
f(R, t)=n_{0} e^{-4 \beta t} Z^{-1}\left(R_{\max }^{0}-R e^{-\beta t}\right)^{d},
$$

where $d=3 R_{\max }^{0} / R_{c}^{0}-4$ and the normalization factor $Z=(1+d)^{-1}\left(R_{\max }^{0}\right)^{1+d}$. As $f(R, t)$ has to remain finite everywhere in the interval $\left\{0, R_{\max }\right\}$, the power coefficient $d$ is required to be positive, thus defining a one-parametric family of self-similar solutions. This is totally different from the one-parametric family of solutions obtained in the classical theory of OR (see Ref. [31], and references therein). The examples of CRDs [functions $g(R)$ ] from the one-parametric family described by Eq. (9) are presented in Fig. 1.

It is worth considering the extreme opposite case $\lambda \ll R$, in which the main equation of classical Wagner theory of OR can be recovered within our approach based on the calculation of the return probability $P(R, \lambda)$. In the limit $\lambda \ll$ $R$, the molecules emitted from the cluster and scattered outward after the first collision still have a very high probability to return to this cluster due to subsequent collisions. This makes the collision-based calculation of $P(R, \lambda)$ difficult, as the multiple-collision terms cannot be neglected. We show that in this case one can calculate the escape probability $1-P(R, \lambda)$ using an electrostatic analogy.

Consider a stationary point source $S$ emitting molecules with constant rate $W$ and located at a distance $\xi$ from the sphere $A$ of radius $R$, which absorbs molecules and does not reemit them. A probability for a molecule emitted from $S$ to travel to infinity can be associated with the ratio $W_{\text {inf }} / W$, where $W_{\text {inf }}$ is a steady-state molecular flux crossing the surface of an infinitely large encompassing sphere. As the steady-state concentration $N(r)$ obeys Laplace's equation $\Delta N(r)=0$, one can use electrostatic analogy to calculate molecular flux $W_{\text {inf }}$ by replacing $N(r)$ with the electrostatic potential, molecular flux with the electric field flux, and the molecule generation/absorption rate with a positive/negative electric charge. For illustration purposes, we assume that the sphere $A$ absorbs every molecule touching its surface, so that molecule concentration $N(r=$ $R$ ) (electrostatic potential) at its surface is 0 . Then the source $S$ and the sphere $A$ can be replaced with a point charge $q=W$ and an earthed metallic sphere of radius $R$, respectively. The flux $W_{\text {int }}$ can then be calculated using Gauss' theorem $W_{\text {int }} \propto(q+Q)$, where $Q$ is the charge induced on the earthed sphere by the point charge $q$. The value of $Q$ is known to be $Q=-q R /(R+\xi)$, suggesting that the escape probability for molecules emitted from $S$ is equal to $\xi /(R+\xi)$.

If we now associate the sphere $A$ with the molecular cluster, and the source $S$ with the point at which a molecule emitted from this cluster has its first collision, then the overall escape probability can be determined as [32]

$$
1-P(R, \lambda) \simeq \frac{1}{\lambda} \int_{0}^{\infty} \frac{\xi \exp (-\xi / \lambda)}{R+\xi} d \xi=\frac{\lambda}{R} \int_{0}^{\infty} \frac{x \exp (-x)}{1+\lambda x / R} d x=\frac{\lambda}{R} \Phi(\lambda / R)
$$

By direct calculation, it can be shown that the value of $\Phi(\lambda / R)$ varies by no more than $1 \%$ of its asymptotic value $\Phi(\lambda / R \simeq 0)=1$ at all $\lambda / R \leq 0.005$, allowing one to use an approximation for the return probability $P(R, \lambda) \simeq 1-$ $\frac{\lambda}{R}$. Substituting this into Eq. (3) and then into Eq. (2), one obtains the equation characteristic for interface-limited OR in Wagner theory [11]

$$
\frac{d R}{d t}=K \nu\left(\frac{\lambda}{R_{c}(t)}-\frac{\lambda}{R}\right), \quad R_{c}(t)=\frac{\left\langle R^{2}\right\rangle}{\langle R\rangle} .
$$

The analytical results given by Eq. (9) are verified by numerically solving a discrete version of Eq. (6) to describe the time evolution of clusters with radii distributed in the range $\left\{R_{\min }=0.01, R_{\max }=10\right\}$. Numerical solution of differential equations was performed using a standard finite difference scheme. Initially, the system contains $10^{7}$ equations, each corresponding to a single cluster. This number rapidly decreases in the course of simulations because of the disappearance of small clusters and an increasing range of cluster radii, which results in fluctuations in the cluster size distribution function. To cover all the time range of interest, these fluctuations were removed by increasing the number of clusters (hence, equations) at two intermediate time moments $t_{1}=50$ and $t_{2}=90$ (curves 3 and 5 in Fig. 2) up to the initial number $\left(10^{7}\right)$ without disturbing the CRDs at these time moments.
Numerical simulations confirmed the existence of the one-parametric family of self-similar solutions given by Eq. (9) with an exponentially growing average cluster size. It was also found that every initial distribution from within this family $(d \geq 0)$ eventually converges to the boxcar distribution $(d=0)$, possibly due to fluctuations in the cluster radius distribution, as in classical OR [33]. These fluctuations result in a spread of initially well-defined parameter $d$, which allows a slightly different value of $d$ to be selected. An example of such a convergence is shown in Fig. 2. The initial distribution with $d=1.0$ (curve 1 in Fig. 2 and curve 2 in Fig. 1) remains almost intact (curves 2-4) for the time period during which the average cluster radius increases exponentially (see inset in Fig. 2) by about an order of magnitude. Then, within the time period $70<t<110$, the distribution transforms (curves 5 and 6), simultaneously decreasing the rate of exponential growth of the average cluster radius. The resulting boxcar distribution remains then stable during the whole course of the simulation. Any other initial distribution also converges to the boxcar distribution, but in its own way. Understanding the details of such convergences requires further studies.

This entirely new regime of steady-state Ostwald ripening for the extreme case $\Lambda \gg \lambda \gg R$ is indicative of the strong dependence of coarsening phenomena upon the 


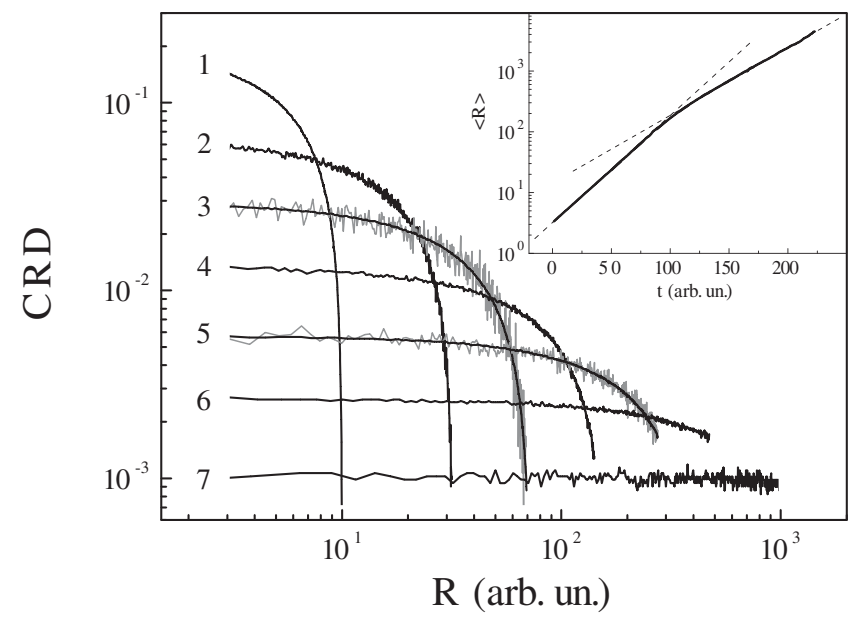

FIG. 2. Simulation results of CRDs at different times: 1,$0 ; 2$, $30 ; 3,50 ; 4,70 ; 5,90 ; 6,110 ; 7,140$. Black curves 3 and 5 replace noisy distributions shown by gray curves - see text for details. The inset shows the time dependence of the average cluster radius, in which one exponential law changes into another upon transformation of the CRD.

regime of molecular transport. Deviations from the classical theory of OR should be anticipated at any $\lambda$ values comparable to or exceeding the cluster radii. These deviations would be more pronounced for the small-sized clusters, resulting in an increase of their fraction in the cluster size distribution compared to that in LSW theory. The results we have presented open the possibility of controlling the shape of a cluster size distribution and the rate of the coarsening process by varying $\lambda$ through a buffer gas density. They might be useful for understanding coarsening phenomena in new classes of systems such as vapor synthesis of nanostructures, clouds in the upper atmosphere, and molecular interstellar clouds.

The author thanks A.P. Sutton and D. G. Pettifor for useful and stimulating discussions and for the opportunity to use Materials Modelling Laboratory facilities. Financial support from the Toppan Printing Co. (Tokyo, Japan) is gratefully acknowledged.

[1] W.Z. Ostwald, Z. Phys. Chem. 37, 385 (1901).

[2] P. W. Voorhees, Annu. Rev. Mater. Sci. 22, 197 (1992).

[3] D. A. Porter and K. E. Easterling, Phase Transformations in Metals and Alloys (Chapman and Hall, New York, 1992), 2nd ed.

[4] M. Zinke-Allmang, L.C. Feldman, and M.H. Grabow, Surf. Sci. Rep. 16, 377 (1992).

[5] P. Taylor, Adv. Colloid Interface Sci. 106, 261 (2003).

[6] W. N. Bartelt, C. R. Theis, and M. Tromp, Phys. Rev. B 54, 11741 (1996).

[7] I. Goldfarb and G. A.D. Briggs, in Recent Research Developments in Materials Science (Research Signpost, Trivandrum, India, 1998), Vol. 1, p. 189.

[8] N. V. Mantzaris, Chem. Eng. Sci. 60, 4749 (2005).
[9] M. Lifshitz and V. V. Slyozov, Zh. Eksp. Teor. Fiz. 35, 479 (1958) [Sov. Phys. JETP 8, 331 (1959)].

[10] M. Lifshitz and V. V. Slezov, J. Phys. Chem. Solids 19, 35 (1961).

[11] C. Z. Wagner, Electrochem. 65, 581 (1961).

[12] B. K. Chakraverty, J. Phys. Chem. Solids 28, 2401 (1967).

[13] C. V. Thomson, Acta Metall. 36, 2929 (1988).

[14] J. A. Marqusee, J. Chem. Phys. 81, 976 (1984).

[15] N. Akaiwa and P. W. Voorhees, Phys. Rev. E 49, 3860 (1994).

[16] M. K. Chen and P. W. Voorhees, Model. Simul. Mater. Sci. Eng. 1, 591 (1993).

[17] M. Strobel, S. Reiss, and K.-H. Heinig, Nucl. Instrum. Methods Phys. Res., Sect. B 120, 216 (1996).

[18] J. A. Warren and B. T. Murray, Model. Simul. Mater. Sci. Eng. 4, 215 (1996).

[19] M. Shwind and J. Agren, Acta Mater. 49, 3821 (2001).

[20] M. Hanbücken, M. Futamoto, and J. A. Venables, Surf. Sci. 147, 433 (1984).

[21] R.F. Shannon, C. R. Harkless, and S.E. Nagler, Phys. Rev. B 38, 9327 (1988).

[22] S. Arrott, B. Heintich, and S. T. Purcell, in Kinetics of Ordering and Growth on Surfaces, edited by M.G. Lagally (Plenum, New York, 1990).

[23] J. Alkemper, V. A. Snyder, N. Akaiwa, and P. W. Voorhees, Phys. Rev. Lett. 82, 2725 (1999).

[24] W. K. Kim, K. Char, and C. K. Kim, J. Polym. Sci., Part B: Polym. Phys. 38, 3042 (2000).

[25] J.S. Rowlinson and B. Widom, Molecular Theory of Capillarity (Clarendon, Oxford, 1982), Chap. 2, pp. 2547.

[26] C. Cercignani, Rarefied Gas Dynamics from Basic Concepts to Actual Calculations (Cambridge University Press, Cambridge, England, 2000).

[27] G. P. Dransfield, K. A. Fothergill, and T. A. Egerton, in Euro Ceramics, edited by G. Dewith, R. A. Terpstra, and R.M. Metsalaar (Elsevier Applied Science, London, 1989), Vol. 1, pp. 275-279; K. Deller, M. Ettlinger, S. Fisher, and R. Weinland, Eur. Cosmet. 57, 391 (1991); J. F. Edd, U.S. Patent No. 4022872 (to Alcoa); S. R. Blackburn, T. A. Egerton, and A.G. Jones, Br. Ceram. Proc. 47, 87 (1991); P. Fauchais, E. Boudrin, J.F. Caudert, and R. McPherson, in Topics in Current Chemistry, edited by S. Venprek and M. Venoguplan (Springer, Berlin, 1983), Vol. 107, pp. 59-183.

[28] F. Qia, T. A. Egerton, and I. L. Cooper, J. Eur. Ceram. Soc. 26, 37 (2006).

[29] J. G. McLean, B. Krishnamachari, D. R. Peale, E. Chason, J.P. Sethna, and B.H. Cooper, Phys. Rev. B 55, 1811 (1997).

[30] W. W. Mullins, J. Appl. Phys. 59, 1341 (1986).

[31] B. Giron, B. Meerson, and P. V. Sasorov, Phys. Rev. E 58, 4213 (1998).

[32] We ignore the contribution of molecular collisions in the thin Knudsen layer around the cluster to the overall escape/return probability. At sufficiently small $\lambda / R$ values, this contribution approaches a radius-independent asymptotic value, and taking it into account results in a slight change of the constant $K$ in Eq. (11).

[33] B. Meerson, Phys. Rev. B 60, 3072 (1999). 